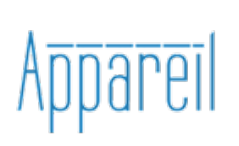

Appareil

Numéro spécial | 2008

La ville dans les sciences humaines

\title{
Perception urbaine, distraction et stratification chez Benjamin, Eisenstein et Vertov
}

Pascal Rousse

\section{OpenEdition}

Journals

Édition électronique

URL : http://journals.openedition.org/appareil/467

DOI : 10.4000/appareil.467

ISSN : 2101-0714

Éditeur

MSH Paris Nord

Référence électronique

Pascal Rousse, « Perception urbaine, distraction et stratification chez Benjamin, Eisenstein et Vertov », Appareil [En ligne], Numéro spécial | 2008, mis en ligne le 30 juin 2008, consulté le 30 juillet 2020.

URL : http://journals.openedition.org/appareil/467 ; DOI : https://doi.org/10.4000/appareil.467

Ce document a été généré automatiquement le 30 juillet 2020

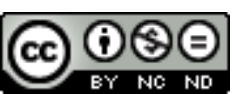

Appareil est mis à disposition selon les termes de la Licence Creative Commons Attribution - Pas d'Utilisation Commerciale - Pas de Modification 4.0 International. 


\title{
Perception urbaine, distraction et stratification chez Benjamin, Eisenstein et Vertov
}

\author{
Pascal Rousse
}

La spatialité (Raumlichkeit) peut être la projection de l'extension (Ausdehnung) de l'appareil psychique. Aucune autre dérivation n'est plausible. À la place des conditions a priori de Kant, notre appareil psychique, la psyché est étendue (ausgedehnt), je ne sais rien de plus à ce sujet ${ }^{1}$.

1 Si la présence massive de la ville à l'écran semble attester que l'urbain est le substrat historique et imaginaire du cinéma - à tel point que, pour le Néoréalisme et pour la Nouvelle vague, mais aussi pour $\mathrm{Kracauer}^{2}$, la rue, espace générique de la ville, se présente comme la matrice même de «l'écriture » du film -, il faudrait se demander quels rapports le cinéma peut nouer avec ce contexte privilégié et en quoi l'appareil cinématographique contribue à en transformer l'espace, tout en se constituant comme sa mémoire. Cela ne peut se jouer sans le spectateur, pour qui chaque film est la présentation d'un milieu entre l'expérience vécue de l'espace concret et l'événement. Walter Benjamin inaugura cette problématique, afin de penser avec la notion de distraction la question de la production de l'espace à l'époque du cinéma, surtout burlesque, d'animation et soviétique. Henri Lefebvre note que Marx a laissé de côté l'ensemble des concepts liés à la reproduction, dont la reproductibilité, mais aussi la question de l'espace de la production et de la reproduction ${ }^{3}$. On peut remarquer que ce sont là justement les champs sur lesquels s'exercera l'essentiel de la pensée de Benjamin.

2 Nous allons voir que la notion de distraction établit un certain rapport entre architecture et cinéma chez Benjamin. Mais nous verrons aussi que le cinéma altère et transforme l'espace concret. Une mise au point s'impose tout d'abord, à la lumière de questions de traduction posées par Jacques Boulet ${ }^{4}$. Le mot dont il est question est en allemand Zerstreuung, que l'on traduit par distraction, ce qui induit en français des confusions avec le divertissement. Or, Zerstreuung ne désigne pas chez Benjamin un état d'esprit susceptible d'un jugement moral, comme le Français l'entend généralement par 
distraction ou divertissement, mais un mode complexe de perception dans l'espace social de la ville. Ce mode de perception implique le contact, dont Freud affirme dans Inhibition, symptôme et angoisse (1925), qu'il est le principe même d'Eros. Voici ce qu'écrit Freud:

Si l'on se pose la question de savoir pourquoi l'évitement du toucher, du contact, de la contamination, joue un aussi grand rôle dans la névrose et pourquoi il devient le contenu de systèmes aussi compliqués, on trouve comme réponse que le toucher, le contact corporel, est le but premier de l'investissement d'objet, aussi bien agressif que tendre. L'Eros veut le toucher car il aspire à l'union, à la suppression des frontières spatiales entre le moi et l'objet aimé. Mais la destruction aussi, qui avant l'invention de l'arme à distance ne pouvait s'effectuer que dans la proximité, présuppose nécessairement le toucher corporel, porter la main sur autrui ${ }^{5}$.

3 Ce passage capital s'articule avec la notion d'état de détresse du nourrisson (Hilflosigkeit), c'est-à-dire l'inachèvement de l'être humain et la séparation d'avec l'Autre, qui le constituent. Georg Simmel, dans Les grandes villes et la vie de l'esprit (1903), montrait déjà comment le citadin $\mathrm{du} \mathrm{xx}^{\mathrm{e}}$ siècle, soumis à des contacts permanents, développe des réflexes de mise à distance, précisément par des formes d'inattention à autrui et aux choses destinées à prévenir la violence potentielle d'une trop fréquente proximité6. En revanche, Elias Canetti montrera dans Masse et puissance (1959) que le phénomène de la masse se caractérise précisément par le retournement transgressif de la phobie du contact? ${ }^{7}$.

4 Ainsi, la notion de Zerstreuung développe une dialectique entre contact et séparation, Eros et Thanatos, choc et dispersion, afin de penser le rapport entre le phénomène des masses urbaines, la technique et la politique : il s'agit de montrer en quoi le cinéma constitue l'enjeu d'une réforme de la sensibilité entre la reproduction régressive des masses comme telles en masses fermées ou la constitution de masses ouvertes capables de surmonter et de transformer politiquement l'espace donné. Cela dit, pour la commodité du renvoi aux traductions existantes je garderai le terme de distraction dans cet exposé, à entendre donc en un sens soigneusement distinct, voire au contraire du divertissement. Mais je reviendrai sur les notions d'inattention et de disruption que Jacques Boulet propose pour traduire le mot Zerstreuung.

5 La perception distraite, donc, fournit au cinéma le substrat psychophysique sur lequel vont s'étayer ses effets perceptifs et cognitifs pour le spectateur. Dans toutes les versions du texte sur lequel je m'appuie: L'Euvre d'art à l'époque de sa reproductibilité technique $^{8}$, Benjamin met d'abord en place dialectiquement les concepts de reproductibilité technique et d'authenticité, de proximité et d'aura, de valeur de culte et de valeur d'exposition, de test et de jeu, de totalité et de montage. Ensuite, la présentation de la notion de distraction, anticipée par le dadaïsme comme provocation ou diversion (Ablenkung), succède à la comparaison de l'architecture, de l'épopée et du cinéma en tant qu'ils s'offrent à un mode collectif, simultané et inconscient de perception, par opposition à la peinture qui requiert une attitude contemplative. Puis, la perception tactile de l'architecture est développée : la perception tactile constitue le substrat de la perception optique, lesquelles sont alors coordonnées par l'habitude, par opposition à l'attitude séparée et focalisée de l'amateur de monuments. L'habitude est une mémoire psychophysique, l'incorporation de savoirs-faire, de capacités, acquis par une heuristique technique et permettant la répétition, la reproduction de ces habiletés au niveau du réflexe, libérant l'attention consciente : toutes les techniques, de la chasse à la rhétorique, en passant par la guerre et l'art supposent une stratification incorporée 
de savoirs, de gestes, de rythmes qui étayent la pensée et donnent consistance au langage.

6 Le concept de stratification vient du latin des alchimistes (stratificatio, onis) et est passé ainsi à la chimie (1620). Du latin stratum, «chose étendue ", dérivé par substantivation de sternere, "étendre ». L'évolution du terme est intéressante puisque l'on passe en latin d'un sens dynamique, temporel, au sens statique et spatial du résultat, tandis que le sens moderne du paradigme partira de la chose observable, la strate en tant que couche sédimentaire, objet d'une sémiologie d'où l'on induira une reconstitution du passé et un récit de la préhistoire. Le terme de strate apparaît en 1805, venant de strata (1765) désignant en géologie chacune des couches de matériaux qui constituent un terrain, en particulier sédimentaire (assise, banc, couche, lit), renvoyant alors au processus géologique par lequel ces matériaux se sont ainsi disposés (stratification concordante, discordante, entrecroisée, horizontale, inclinée). On parlera ensuite, en biologie, des strates de cellules d'un tissu et en psychologie des strates de souvenirs. Les concepts de strate et de stratification s'emploient aussi en un sens précis en sociologie. C'est donc un paradigme fécond, transversal, permettant une analyse et une compréhension spatio-temporelle des phénomènes. Freud l'emploie dès les Études sur l'hystérie (1895), où la métaphore archéologique apparaît et persistera dans l'étude sur la Gradiva de Jensen (1907) et dans l'image de Rome de Malaise dans la civilisation (1929); elle prendra et gardera un sens à la fois topique et dynamique, mais aussi psychique et sociologique ${ }^{9}$. La stratification, en effet, n'est pas statique, elle implique toujours du mouvement plus ou moins rapide, des durées plus ou moins étendues, des déplacements, des glissements, des effondrements, des remaniements et des surgissements, bref une plasticité, comme la mémoire, mais aussi un espace d'obstacles et de passages qui fait tout le bonheur des récits et des films d'aventures. À la stratification géologique immémoriale répond la stratification urbaine, et l'archéologie nous fait passer de l'une à l'autre.

7 Le paradigme de la stratification (Schichtung) chez Freud lie le concept de stades du développement du Moi selon Ferenczi à celui des couches (Schichte) de la mémoire dans l'appareil psychique. Il permet en outre d'articuler avec une grande économie l'ontogenèse et la phylogenèse, c'est-à-dire l'individuation psychique et l'individuation collective des groupes comme de l'espèce humaine. La conception globale du temps est essentiellement darwinienne chez Freud, d'un Darwin donnant pour preuve de l'évolution sa reproduction et récapitulation condensée dans le développement de l'embryon, d'une part, et, d'autre part, la survivance des stades antérieurs à un stade plus récent dans des traces anatomiques non fonctionnelles, voire dysfonctionnelles ou pathologiques ${ }^{10}$. Cela éclaire, chez Benjamin, comme nous allons le voir, les notions d'inconscient visuel et de perception distraite. Précisons tout de suite que l'inconscient visuel peut ainsi être situé tout autant dans la ville elle-même, comme dans la Pompéi de Gradiva ou la Rome de Freud, que dans la mémoire de l'être singulier ${ }^{11}$. La ville recèle son propre inconscient visuel, où s'inscrivent jusque dans les affiches et les enseignes les traces de la mémoire et de l'imaginaire collectif, et c'est de la rencontre, du choc avec le psychisme de l'être affecté que surgissent des images. C'est le modèle du geste disruptif du flâneur, en ce qu'il se livre entièrement à ce processus : tels Baudelaire et les surréalistes, mais aussi Joyce ${ }^{12}$. C'est aussi pour Benjamin une méthode de déchiffrement de l'espace social et symbolique. 
8 La distraction est donc un mode inconscient de perception à l'état de veille ; il est réellement inconscient en ce qu'il est autonome par rapport à la conscience et remplit précisément des fonctions d'ordre réflexe là où la conscience est devenue inopérante, voire gênante. La distraction s'exerce dans des situations d'hétérogénéité, de rapidité et de dispersion des stimuli, où une décision rapide est nécessaire et où seule la capacité acquise d'accomplir certains gestes permet d'agir. Mais la distraction s'impose également dans des chaînes opératoires complexes et répétitives finement réglées et synchronisées, contrôlées par le test, dans lesquelles le moindre décalage produit des dérèglements en chaînes. Chaplin l'a merveilleusement mis à l'écran dans Les Temps modernes (1936). Le paradoxe étant que la perception distraite, on le voit, demande alors le renforcement d'une certaine forme d'attention des plus archaïques : le qui-vive permanent du chasseur, de la sentinelle, mais aussi de l'espion et du détective. Il faut donc une attention à la fois flottante, non focalisée et prête à anticiper et à saisir à tout moment l'occasion propice, le kaïros. C'est un état d'attente ouverte et indéterminée dans un espace de possibilités multiples et indéfinies. L'autre paradoxe est que cela implique l'habitude, c'est-à-dire la capacité d'habiter un milieu ou, en d'autres termes, une attention simultanée à tout ce qui advient.

Depuis Maine de Biran, en passant par Hegel, l'habitude ${ }^{13}$ permet d'analyser la relation dynamique du physique et du psychique, mais aussi de l'individuel et du collectif, et sert à Benjamin de fondement pour construire la notion de distraction. La question de l'habitude se situe au croisement de la philosophie et de l'anthropologie, de l'éthique et de la psychologie : c'est le point où se réfléchit le problème du retrait de l'instinct au bénéfice d'un système nerveux ouvert, dont le cerveau est l'organe de coordination, et que s'enracine la fonction symbolique articulée à la technique. L'état de détresse du nourrisson est la marque de cet inachèvement constitutif et le lieu même où se lient l'affect et le symbolique. La mémoire comme fonction inconsciente, entre soma et psyché, l'une des découvertes majeures de Freud, est étroitement liée à l'habitude et à ses écarts, lapsus et actes manqués. Benjamin en porte la problématique à l'échelle du collectif ou de la masse et va plus loin que Freud en cela, lequel eut à réaffirmer, contre la notion d'inconscient collectif de Jung, l'ancrage de la psychanalyse dans le sujet et l'expérience clinique. Benjamin, dans L'Euvre d'art à l'époque de sa reproductibilité technique répond manifestement aux problèmes de l'approche freudienne du phénomène des masses et cela grâce au concept éminemment freudien d'appareil (Apparat, du latin apparatus), qu'il rapporte à son modèle technique : l'ensemble des appareils optiques permettant de transformer automatiquement la vision ${ }^{14}$. L'importance de la distraction dans la ville moderne est l'indice qu'une nouvelle mémoire collective s'élabore, qui se substitue au mythe, au rituel, à la coutume et à la narration, c'est-à-dire à la tradition, face aux conditions de danger mortel qui accompagnent la mutation anthropologique en cours depuis la révolution industrielle. L'appareil, en tant qu'il articule la technique et le symbolique, permettant d'opérer cette transformation collective de la mémoire, est le cinéma. Le cinéma substitue à l'espace traumatique de la ville industrielle l'espace de jeu (Spielraum) de la mise en scène et du montage, selon la structure du fort/da, de la disparition et de l'apparition, présentée par Freud dans Au-delà du principe de plaisir (1920) ; ainsi, écrit Benjamin, le jeu est-il « la transformation d'une expérience bouleversante en habitude ${ }^{15}$. »

L'échelle des forces de concentration et de décharge, de destruction et de retour à l'inertie absolue déclenchées par le processus industriel est inhumaine, traumatique : 
s'y adapter simplement voudrait littéralement dire disparaître, comme le montre la mutation de la guerre en destruction totale. L'être humain ne s'adapte pas seulement, mais modifie son environnement techniquement et symboliquement ; il lui faut donc, à chaque fois que de nouveaux problèmes de survie sont à résoudre et atteignent un certain seuil, inventer un monde, un milieu doté d'une enveloppe, à partir d'un ensemble de dispositifs régis et articulés par un appareil. Ainsi, si l'on suit chez LeroiGourhan le passage de l'esthétique fonctionnelle, à l'intégration architecturale du monde et à l'espace social, on peut dire que certaines villes ou cités-États, constituèrent dans l'antiquité des formes nécessaires, articulant technique et symbolique, permettant de connecter et de coordonner un ensemble de dispositifs formant ce que l'on appelle une civilisation ${ }^{16}$. L'habitat est, selon Benjamin, la forme la plus ancienne de la sensibilité collective, innervant tactilement les corps et ménageant l'espacement entre constance et variation, entre espace et temps. L'architecture élabore et fixe les schémas d'organisation de l'habitat, selon l'appareillage technique et symbolique des communautés entre la circularité du foyer et la ligne droite du cordeau. L'architecture grecque classique est un des premiers exemples, dans le temple, de dissociation marquée entre fonction porteuse et séparation du dedans et du dehors, ainsi que d'équivalence entre le haut et le bas. Le classicisme serait ainsi la consécration, dans l'usage harmonique de la réversibilité spatiale, des valeurs d'isonomie et d'autonomie que garantissent les lois de la Cité. Le problème de la modernité est d'articuler l'aspiration à l'autonomie et à l'égalité avec la multiplicité, la dispersion, l'hétérogénéité irréductibles des forces à l'œuvre dans l'espace urbain de la révolution industrielle. L'architecture moderne visera à intensifier les valeurs omnidirectionnelles incorporées dans la réversibilité spatiale, où le haut et le bas sont absolument symétriques et où la paroi n'est plus qu'un écran flottant ou une membrane entièrement dissociée de l'ossature: l'idée de promenade architecturale chez Le Corbusier est exactement contemporaine de l'invention par Murnau de la caméra mobile, dans Le Dernier des hommes, en 1923. Celle-ci n'est pas alors synonyme de caméra subjective, mais au contraire de dépossession du sujet : la caméra mobile de Murnau, comme la promenade architecturale, plonge la perception optique dans la perception tactile. C'est ce que Le Corbusier appelle l'intention motrice, dont le plan d'architecture devient la condensation ouverte ${ }^{17}$.

11 Mais comment penser l'écart entre expérience et distraction? Si l'expérience suppose la conscience, la perception distraite en revanche suppose une éclipse de la conscience. La perception distraite est une dispersion, une dissémination pour la conscience, dont la fonction de pare-excitation est mise à mal par la saturation traumatique des chocs dans l'expérience vécue (Erlebnis) et constitue cependant un mode nouveau d'orientation sur le plan de l'inconscient: un réseau de coordonnées psychophysiques sur lesquelles fait fond le cinéma en construisant l'ellipse du montage, comme cette courbure spatiotemporelle tendue entre le double foyer de deux plans. C'est la texture psychique du collectif dans laquelle l'œuvre d'art, y compris architecturale, est absorbée à l'état d'image reproductible et transformée. L'architecture, qui distingue le dedans et le dehors et construit des seuils entre l'un et l'autre par l'invention et la mémorisation de schèmes ambulatoires, articule perception tactile et optique. C'est alors une idée de l'inconscient qui prend le relais, lorsqu'on est conduit à admettre que le corps sent, pense et dirige. Ces intermittences entre le corps et l'esprit, entre langage et image, entre l'être et l'espace sont le matériau du cinéma. Elles croisent, dans le texte de Benjamin, la distinction entre le monumental, en tant qu'architecture qui se 
donne à voir et tient en respect le regard de celui qui lève les yeux sur l'édifice, d'une part, et d'autre part, les espaces du collectif, espaces voués à la perception distraite et ouverts par la pénétration de l'œil de la caméra, cher à Vertov, à l'action dis-locatrice, disruptive et transformatrice des masses.

Tenir en respect, c'est tenir en place sous le regard et faire lever les yeux vers soi : c'est, à la limite, le rapport entre dominant et dominé, forme et matière, contenu et forme, sous-jacent au phénomène de l'aura. Être tenu en respect, c'est être arrêté et retenu par un regard, virtuellement armé. À cela s'oppose une esthétique polyfocale du choc et du fragment, dont l'enjeu artistique est la mise à distance par l'accoutumance et l'habitude de l'expérience vécue du choc, et non l'exaltation du choc par une représentation persuasive de l'expérience. La reproduction technique est, de plus, production de fragments et d'un monde par le montage, et non plus évocation contemplative d'une totalité perdue, laquelle est précisément, pour Benjamin, le divertissement, la fuite devant l'urgence politique. L'aura est une trame d'espacetemps, un rapport du proche et du lointain, où le lointain est l'inapprochable ; c'est la formule métaphysique du pouvoir. Si le lointain domine dans la définition de l'aura, c'est surtout en tant qu'il est pris en une apparition unique et qui se donne pour naturelle, dans la temporalité suspendue, la durée d'une contemplation du paysage, sans aucune médiation c'est-à-dire dans la plus complète solitude et sans autre fin qu'elle-même. C'est en somme l'archétype de l'expérience du beau selon Kant, laquelle précèderait toute élaboration artistique, toute tekhnê. Dans la distraction, en revanche, c'est le proche qui domine, mais d'abord dans l'immersion d'une proximité excessive des processus techniques déchaînés de la seconde nature industrielle. C'est l'expérience vécue du choc, qui fait éclater et dissout l'identité du sujet.

Tout le problème est de faire droit aux valeurs, aux teneurs de vérité, enfermées dans ces deux extrêmes, dans un nouveau rapport du proche et du lointain. Comment, en effet, par-delà toute fantasmagorie, atteindre certains lointains, se laisser toucher par le passé le plus ancien, et, en même temps, mettre à distance le trop proche, le choc qui paralyse toute réflexion et nous soumet sans recours à un donné parfois insoutenable? Car il y va des rapports entre l'individuel et le collectif qui déterminent les conditions de possibilité de la pensée, de l'action et du faire. On voit bien toute la différence entre l'élaboration artistique du montage au cinéma, dont la finalité est de recréer de l'espacement dans le chaos des sensations, et l'effet des flux d'images télévisuelles de catastrophes qui visent à anéantir la pensée par les sentiments de l'impuissance et du ressentiment et fait de la souffrance d'autrui un divertissement. Le montage est polyfocal, il produit et apparie des espaces-temps qui incorporent pour la conscience et rendent pensable, en la dédoublant techniquement, la perception distraite.

Le regard distrait, celui du flâneur en particulier, produit inconsciemment des impressions fortuites, des aperçus, tout autant qu'il absorbe des chocs, lesquels peuvent donc être d'abord infinitésimaux. Le flâneur, pour autant, agit méthodiquement, par répétition et variation, allers et retours, dans la mesure où il procède d'une attitude et d'une démarche liées au milieu urbain, qu'il s'agit de s'approprier et d'habiter. Ces coups d'œil involontaires fournissent un matériau mémoriel, qui semble oublié aussitôt, lequel constituera pourtant l'inconscient productif : notamment pour le poète, le peintre de la vie moderne et le caricaturiste. Par le biais de la Psychopathologie de la vie quotidienne (1901), étroitement liée à L'Interprétation des rêves (1900) chez Freud ${ }^{18}$, Benjamin associe l'inconscient visuel et l'inconscient pulsionnel. L'important est le lien 
fortement établi entre oubli et mémoire, d'une part, et entre l'affect et le banal, d'autre part. En effet, comme l'a montré Freud, c'est précisément le détail quelconque, l'objet anodin, le lieu commun qui offrent aux pensées refoulées l'occasion de tromper la censure de la conscience, dans le lapsus, le mot d'esprit ou le rêve. Nul doute que ces processus ne motivent l'intérêt dramatique pour le détail fortuit, l'objet familier, qui acquiert son pouvoir de fascination non seulement de par sa fonction-clé dans une intrigue, mais aussi et plus encore du déplacement qui s'opère ainsi et s'offre à la condensation et à la projection fantasmatique du spectateur. La ville abonde, bien entendu, particulièrement dans l'art des devantures, de tels objets jusqu'au vertige et à la saturation - et l'on pense alors à M le maudit de Fritz Lang (1932), où la vitrine se présente comme une métaphore de l'écran, ce qui fait dialectiquement de l'écran une métaphore de la vitrine, comme on le voit déjà dans Le ballet mécanique de Fernand Léger et Dudley Murphy (1924). Chez Eisenstein, en particulier, l'équivalent de cet investissement fantasmatique dans le détail quelconque et altéré par l'affect est le gros plan. La vivacité du regard du flâneur, sous-tendue par la lenteur de son allure, semble ainsi être la condition de sa constitution en trace mnésique productive pour l'inconscient, mais aussi de la maitrise du choc; elle trouve son équivalent rythmique dans le vers disruptif de Baudelaire, si finement perçu par Benjamin, puis dans le montage musical de Dziga Vertov.

Le cinéma est ainsi un mode de production de l'espace et du temps; il opère la mise en œuvre de la mémoire et de la puissance de transformation de nos déplacements physiques et psychiques, mais il contribue aussi puissamment à leur remaniement. L'appareil cinématographique pro-duit, en la donnant à voir, la production de l'espace elle-même, en même temps qu'il invente des modèles de sa transformation. Le mouvement nécessairement discontinu, elliptique, entre les plans, le rapprochement des temps et des lieux, sont l'affaire du montage, lequel joue ainsi de la mémoire et de l'affect. L'affect élit et relie en les remaniant les couches de la mémoire et ouvre la voie à l'irruption figurale de manifestations de l'inconscient. La figure du flâneur chez Baudelaire, les surréalistes Joyce et Benjamin, nous conduit à travers les strates d'une construction du regard et du geste, qui trouvera dans le cinéma son mode d'écriture et de pensée : travail d'invention de la trace. Ainsi, la perception distraite implique la stratification de la mémoire et de l'inconscient dans un imaginaire actif qui innerve l'être singulier quelconque, issu des masses.

L'expérience vécue, celle du singulier, est d'être dans l'espace et dans le lieu, inclus et enveloppé, voire enfermé. Or, selon Benjamin, la masse comprend en elle le monument par l'imaginaire; en quelque sorte, elle le digère et donc le maîtrise. Selon Adrienne Monnier, inspiratrice de Benjamin, la masse reconnaît et s'approprie ce qu'elle a engendré. Grâce à la reproduction des œuvres et, en particulier, la diffusion des photographies d'œuvres architecturales, ce pouvoir des masses acquiert son support et ses titres ${ }^{19}$. La carte postale, en particulier, que nous retrouvons dans Les Carabiniers de Godard (1963), est bien l'insigne du pouvoir qu'a désormais le quelconque, en tant qu'être singulier de masse, d'absorber le monument et de surmonter son inclusion spatiale. De même et plus encore au cinéma, où tant d'expériences d'évasion et de voyage sont procurées au spectateur, lesquelles peuvent le conduire à la connaissance de soi et au réveil de la raison par la transformation de l'affect ${ }^{20}$. Si le spectateur consent à abandonner le privilège de la flânerie en s'asseyant dans la salle obscure, il en attend le bénéfice d'une expérience (Erfahrung) qui tend à une simultanéité augmentée, comme le veut Koulechov, et lui restitue un espace métonymique jamais vu qui ouvrira 
sa mémoire à ses propres chemins. Il y a donc un rapport de disproportion disruptive entre la durée d'un film et la capacité du cinéma à monter des espaces-temps multiples et hétérogènes, qui est un pouvoir de condensation hors de portée du psychisme nu. Ainsi, écrit Jean-François Lyotard dans Discours, Figure : « la relation au support définit la nature du signe et par là même implique une certaine sorte de relation entre ses trois pôles ", que sont la figure, le support et le sens. Jacques Boulet précise que ce rapport définit à chaque fois un mode d'espacement et d'écart ${ }^{21}$. Ce qui est artistique dans un film, c'est une forme de conquête de l'ubiquité dont le pouvoir de condensation est comparable à celui de la grande ville, mais sur un plan ek-statique, comme l'affirme Eisenstein, où le spectateur sort de soi et peut accéder à une expérience qui excède son enfermement dans un point de vue ${ }^{22}$. L'œil de la caméra n'est le point de vue de personne et le montage articule les prises de vues de l'appareil, dont la surface de régulation n'est plus la fenêtre ouverte mais l'écran, lequel est le principe architectonique du plan comme de la salle de cinéma. L'écran de cinéma se définit par l'ouverture de ses bords au " hors-champ » et non plus, comme dans le tableau, par la fermeture de son cadre. Ainsi, Benjamin peut-il affirmer que le cinéma offre bien plus que des figures d'identification, mais aussi une expérience d'altération et d'altérité capable de provoquer le transfert des affects collectifs, par-delà la katharsis.

Quel rapport dans le matériau et dans la forme avec l'architecture? Avec le burlesque ou le film d'animation, c'est lorsque la stabilité même de l'habitat humain est menacée que le processus du film atteint sa plus grande puissance sur l'imagination du spectateur qui, loin de s'endormir, redouble d'attention et de tension comme s'il y allait de lui-même, de sa propre position fondamentale dans le monde et de sa propre consistance existentielle. C'est le cas dans La Ruée vers l'or, de Chaplin (1925), où Charlot est pris dans une cabane qui bascule au bord de l'abîme, et chez Buster Keaton, bien sûr, mais aussi chez le premier Walt Disney, avant Bambi (1942). L'accomplissement même du processus burlesque est l'anéantissement de toutes les assises de l'inhérence spatiale dont l'étape ultime est la destruction fascinante, catastrophique et inéluctable de tout abri, de toute construction. Mais avant cela, les espaces construits sont les lieux privilégiés des passages, poursuites, retournements, acrobaties et jeux de cache-cache. Dans Le Journal de Gloumov d'Eisenstein (1923), la séquence : entrée dans le bâtiment, ascension de la tour, vue d'en haut, saut dans le vide, rattrapage par un avion, atterrissage dans une automobile est statistiquement la séquence type commune au film burlesque et au film d'action dès les débuts du cinéma, fondée sur les pôles constitutifs du champ de l'expérience et de l'imaginaire urbains. Ce petit film d'Eisenstein, destiné à être inséré dans une mise en scène théâtrale, Le Sage, constitue une double parodie: de l'américanisme cinématographique de Koulechov, d'une part, lequel sert, d'autre part, à parodier le «Ciné-journal» du groupe de Dziga Vertov; celui-ci avait été nommé pour encadrer ces jeunes gens et avait dû jeter l'éponge devant leur résistance à ses thèses sur le "Cinéma-vérité » (Kino-pravda). Mais c'est en même temps un témoin des recherches et des acquis du formalisme russe dans le cinéma.

Dans L'Homme à la caméra, de Dziga Vertov (1929), le passage du rapport architectonique entre l'œil de la caméra (Kino-glaz) et les masses urbaines au caractère architectonique de l'écran dans la salle de cinéma est remarquable. Dans le final du film, en particulier, à partir du moment où la caméra se présente elle-même (cette séquence est alors un film d'animation), le jeu du montage alterné et réciproque des passages entre dehors (la ville) et dedans (la salle de cinéma) montre avec éloquence que les êtres singuliers se 
reconnaissent eux-mêmes à l'écran, en tant qu'ils émergent du milieu urbain. Le cinéaste récapitule alors l'ensemble du film dans un mouvement d'accélération qui tend à susciter l'expérience cinématographique de la simultanéité. Dziga Vertov situe ainsi l'opération du cinéma par-delà la reproduction des masses en elles-mêmes et pardelà l'identification à une figure de l'Un: l'espace urbain se transforme en scène de l'existence de nouvelles singularités issues des masses révolutionnaires; la surface de l'écran se présente dans le film de Vertov comme l'inter-face de cette transformation.

Tout en polémiquant avec Vertov, Eisenstein reprendra cette conception en la croisant avec la nécessaire dialectique entre fiction et documentaire: il inventera, dans la pensée du montage, une théâtralité et des formes de dramatisation capables d'incorporer l'hétérogénéité anachronique des images. C'est déjà le cas dans une très belle séquence au début de La Grève, d'Eisenstein (1925), intitulée "Chasse à la bête rouge ». Deux ouvriers, meneurs politiques, sont poursuivis par un mouchard de la police : Eisenstein développe l'archéologie du roman policier comme reprise du schème archaïque de la chasse, dans les romans de Fenimore Cooper, par exemple. Le mouchard est associé à la perception optique : la séquence s'ouvre sur le gros plan de son œil qui s'ouvre et se ferme, ensuite son reflet déformé et renversé apparait dans un globe de verre à la vitrine du café où se tiennent ses "proies ", puis il se cache dans une boutique de matériel optique et photographique pour les observer à travers la vitrine. Les ouvriers, en revanche, sont maîtres du terrain: leur aptitude tactique s'adosse justement à la perception distraite du milieu urbain, à l'habitude en tant qu'appropriation de l'espace social. Les mouchards, eux, sont issus d'une "bohème " dépravée, purs produits des contradictions des villes du XIX ${ }^{e}$ siècle. Le film montre, d'ailleurs, le monde ouvrier naissant, circulant entre la campagne où il est encore logé, au début élégiaque de la grève, et la ville. Nous le verrons à la fin («Sixième partie : la liquidation ») pris au piège et massacré par des cosaques dans un logement collectif attenant à l'usine, dont la ressemblance avec une "prison » de Piranèse est marquée par ses passerelles arquées au-dessus de la cour, découpant l'ombre et la lumière et mise en évidence par l'angle de prise de vue à la Rodtchenko. L'ensemble du film se développe dialectiquement entre une comédie des reflets et la dramatisation des corps souffrants des prolétaires, jusqu'à la séquence finale, littéralement panique et traumatique, du massacre systématique des ouvriers mis en parallèle avec des plans documentaires pris dans les abattoirs.

20 De même que la perception distraite des masses rejoint celle de l'enfant qui apprend le décentrement de soi et la dissimulation, Benjamin transpose la méthode psychanalytique sur le plan et à l'échelle des masses, à la suite de Freud. Or, cela nécessite une médiation qui dépasse le cadre de la cure : c'est là que le cinéma joue un rôle crucial indispensable, en tant qu'appareil, comme la psychanalyse ; sa spatialité, celle de l'image-mouvement, appartient aux masses, à l'espace d'action des masses qui ne leur préexiste pas : l'espace d'action des masses urbaines est lui-même discontinu, en transformation, sa temporalité est celle de l'époque des grandes villes et de l'être singulier quelconque qui les habite, dont le cinéma est le mode d'apparition et d'inscription, donc d'existence.

21 Car l'enjeu, chez Eisenstein en particulier, est une articulation de l'espace et du temps selon les paradigmes combinés de l'art de la mémoire ${ }^{23}$ et de la psychanalyse, reliés par le concept de strate et dont l'affect est le fil d'Ariane. Cette conception, assumée dès l'élaboration du montage polyphonique, à partir de La Ligne Générale (1929), 
s'accomplira dans le montage vertical audiovisuel d'Alexandre Nevski (1938) et d'Ivan le Terrible (1944-1946). Ainsi, le montage permet de croiser la simultanéité horizontale d'actions et d'états de choses hétérogènes avec la simultanéité verticale de temporalités hétérogènes, dont la rémanence du passé dans le présent et des futurs inaccomplis, sortes de futurs antérieurs au conditionnel, nourrissent aussi le cinéma contemporain comme celui d'Apichatpong Weerasethakul, Jia Zhang Ke, Béla Tarr, Peter Watkins ou Nicolas Klotz.

\section{BIBLIOGRAPHIE}

Benjamin Andrew, «Ennui et distraction », in Philippe Simay (dir.), Capitales de la modernité. Walter Benjamin et la ville, Paris/Tel-Aviv, Éditions de l'Éclat, 2005.

Boulet Jacques, «Les appareils de l'architecture. La projection orthogonale », in Jean-Louis Déotte (dir.), Appareils et formes de la sensibilité, Paris, L'Harmattan, 2005.

Bruno Giuliana, Atlas of Emotion. Journeys in Arts, Architecture and Film, London/New York, Verso, 2007.

Canetti Elias, Masse et puissance, Paris, Gallimard, 2006.

Conio Gérard, Eisenstein. Le cinéma comme art total, Gollion, Infolio, 2007.

Déotte Jean-Louis, Qu'est-ce qu'un appareil ? Benjamin, Lyotard, Rancière, Paris, L'Harmattan, 2007.

Didi-Huberman Georges, L'Image survivante. Histoire de l'art et temps des fantômes selon Aby Warburg, Paris, Minuit, 2002.

Freud Sigmund, Études sur l'hystérie, Paris, PUF, 1956.

Freud Sigmund, Totem et tabou, Paris, Payot, 1967.

Freud Sigmund, Psychologie des masses et analyse du Moi [1921], in Essais de psychanalyse, Paris, Payot, 1989.

Freud Sigmund, Au-delà du principe de plaisir, Paris, Payot, 1989.

Freud Sigmund, Malaise dans la civilisation, Paris, PUF, 1992.

Freud Sigmund, Inhibition, symptôme et angoisse, Paris, PUF, 1993.

Freud Sigmund, fragment posthume, Londres, 1938, in Pierre Kaufmann (dir.), L'Apport freudien. Éléments pour une encyclopédie de la psychanalyse, Paris, Bordas, 2003.

Freud Sigmund, Euvres complètes. Psychanalyse. Volume IV, 1899-1900. L'interprétation du rêve, Paris, PUF, 2004.

Freud Sigmund, Le Délire et les rêves dans la Gradiva de W. Jensen, Paris, Gallimard, 2006.

Freud Sigmund, Psychopathologie de la vie quotidienne, Paris, Payot, 2006.

Kaufmann Pierre, Psychanalyse et théorie de la culture, Paris, Denoël, 1974. 
Kracauer Siegfried, Theory of Film. The Redemption of Physical Reality, 1940-1960, Princeton, Princeton University Press, 1997.

Lefebvre Henri, La Production de l'espace, Paris, Anthropos, 2000.

Leroi-Gourhan André, Le Geste et la parole. II. La mémoire et les rythmes, chap. XII-XIII, Paris, Albin Michel, 1964.

Liandrat-Guigues Suzanne, Esthétique du mouvement cinématographique, Paris, Klincksieck, 2005.

Lyotard Jean-François, Discours, Figure, Paris, Klincksieck, 1971.

Lyotard Jean-François, Discours, figure, Paris, Klincksieck, 2002.

Meier Alexis, Destins de traces. Pensée et formes de l'architecture "conceptuelle" chez Peter Eisenman, mémoire de doctorat en philosophie, université Paris 8-Vincennes-Saint-Denis, Saint-Denis, 13 juin 2008.

Monnoyer Jean-Maurice, «L'ÆEuvre d'art à l'époque de sa reproduction mécanisée », in Walter Benjamin, Écrits français, Paris, Gallimard, 2006.

Rousse Pascal, « L'Architectonique du montage selon Eisenstein et Benjamin. Architecture temporelle et transformation du lieu », Cadrage.net, octobre 2006, http://cadrage.net/dossier/ architectonique.htm.

Simmel Georg, Les grandes villes et la vie de l'esprit, Paris, Éditions de l'Herne, 2007.

Tackels Bruno, L'Euvre d'art à l'époque de Walter Benjamin. Histoire d'aura, Paris, L'Harmattan, 1999.

Yates Frances A., L'art de la mémoire, Paris Gallimard, 1975.

\section{NOTES}

1. Sigmund Freud, fragment posthume, Londres, 1938, in Pierre Kaufmann (dir.), L'Apport freudien. Éléments pour une encyclopédie de la psychanalyse, Paris, Bordas, 2003, p. 626.

2. Siegfried Kracauer, Theory of Film. The Redemption of Physical Reality, 1940-1960, Princeton, Princeton University Press, 1997.

3. Henri Lefebvre, La Production de l'espace, Paris, Anthropos, 2000.

4. Jacques Boulet, «Quelques notes à propos de W. Benjamin. Suite de mon intervention à la MSH, le 8-5-2003 ", document de travail non publié, Maison des Sciences de l'Homme - Paris Nord, 2003.

5. Sigmund Freud, Inhibition, symptôme et angoisse, Paris, PUF, 1993.

6. Georg Simmel, Les grandes villes et la vie de l'esprit, Paris, Éditions de l'Herne, 2007.

7. Elias Canetti, Masse et puissance, Paris, Gallimard, 2006. Le noyau de ce livre de Canetti est la description d'une émeute ouvrière, à laquelle il participa le 15 juillet 1927 et qui aboutit à l'incendie du palais de justice de Vienne. Étant données les limites de cette étude, précisons que les catégories sociologiques de masse et de flâneur, celle-ci abordée plus loin, ne sont pas employées ici dans une perspective réductionniste, mais comme des catégories limite marquant la crise du sujet.

8. L'Euvre d'art à l'époque de sa reproductibilité technique, reconstitution et traduction de la première version par Bruno Tackels, texte inédit dactylographié, Paris, université de Paris VIIISaint Denis-Vincennes/MSH-Paris Nord, s.d.Voir Bruno Tackels, L'Euvre d'art à l'époque de Walter Benjamin. Histoire d'aura, Paris, L'Harmattan, 1999.

9. Sigmund Freud, Études sur l'hystérie, Paris, PUF, 1956 ; Le Délire et les rêves dans la Gradiva de W. Jensen, Paris, Gallimard, 2006 ; Malaise dans la civilisation, Paris, PUF, 1992. Pour une généalogie du 
paradigme de la stratification chez Freud, voir Pierre Kaufmann, Psychanalyse et théorie de la culture, Paris, Denoël, 1974.

10. Voir Georges Didi-Huberman, L'Image survivante. Histoire de l'art et temps des fantômes selon Aby Warburg, Paris, Minuit, 2002.

11. "Ce destin qui fait naître la ratio dans son autre a son modèle dans le monde de la culture : celui-ci est comme une ville qui contient dans la configuration visible de ses rues, de ses quartiers, une autre configuration, celle qu'elle offrait il y a un siècle, puis une autre encore, chacune reliée aux autres par des aménagements d'urbanisme, tantôt visible, tantôt cachée, de sorte qu'en se promenant dans cette ville qui est le monde de l'esprit, celui-ci expérimente une mobilité fondamentale, non seulement son propre déplacement par rapport à un plan de la cité dont on supposerait qu'il reste immobile et identique à lui-même, mais le déplacement simultané des parties de ce plan qui fait qu'en passant d'un quartier à l'autre, et à la limite, en regardant un même monument, un immeuble apparemment homogène, l'esprit passe d'une ville à une autre ville d'un moment de soi à un autre, et chacun de ces moments ordonne tous les autres autour de soi-même, apparaît comme un foyer autour duquel les autres moments (les autres lieux de la ville auparavant eux-mêmes foyers chacun à son tour) se trouvent déformés, recourbés, méconnaissables. Chaque fois que l'esprit croit prendre de l'ensemble de la ville un intuitus, une vue dépouillée de préjugés, l'acte de regarder produit l'anamorphose de ce qui n'est pas placé au point de bonne vision. » Jean-François Lyotard, Discours, figure, Paris, Klincksieck, 2002, p. 183-184. C'est exactement ce qui se passe dans Ulysse de Joyce. Pour une exploration de l'analogie freudienne de la ville et de l'inconscient dans l'architecture contemporaine, voir Alexis Meier, Destins de traces. Pensée et formes de l'architecture "conceptuelle" chez Peter Eisenman, mémoire de doctorat en philosophie, université Paris 8-Vincennes-Saint-Denis, Saint-Denis, 13 juin 2008, partie II, chap. 2. 3. 1. : « La ville : combinatoire et superpositions, la période excavatrice ».

12. Suzanne Liandrat-Guigues, Esthétique du mouvement cinématographique, Paris, Klincksieck, 2005.

13. Grec hexis, latin habitus, manière d'être, conformation physique/grec ethos, latin consuetudo, habitude, coutume, usage.

14. Voir Jean-Louis Déotte, Qu'est-ce qu'un appareil? Benjamin, Lyotard, Rancière, Paris, L'Harmattan, 2007. Rappelons que les quatre versions du texte de Benjamin se succèdent entre 1936 et 1939 ; les grands textes de Freud sur les phénomènes collectifs commencent dès 1912 avec Totem et tabou (Paris, Payot, 1967) ; Psychologie des masses et analyse du Moi, 1921 (dans Essais de psychanalyse, Paris, Payot, 1989), succède de peu à Au-delà du principe de plaisir (id.), où se met en place la problématique de la pulsion de mort et de la pulsion de vie, en 1920.

15. Walter Benjamin, "Spielung und Spielen », Gesammelte Schriften, Band III, cité par Andrew Benjamin, "Ennui et distraction », dans Philippe Simay (dir.), Capitales de la modernité. Walter Benjamin et la ville, Paris-Tel-Aviv, Éditions de l'Éclat, 2005, p. 148.

16. André Leroi-Gourhan, Le Geste et la parole. II. La mémoire et les rythmes, chap. XII et XIII, Paris, Albin Michel, 1964.

17. Voir Pascal Rousse, «L'Architectonique du montage selon Eisenstein et Benjamin. Architecture temporelle et transformation du lieu», Cadrage.net, octobre 2006, http:// cadrage.net/dossier/architectonique.htm.

18. Sigmund Freud, Psychopathologie de la vie quotidienne, Paris, Payot, 2006 ; Euvres complètes. Psychanalyse. Volume IV, 1899-1900. L'interprétation du rêve, Paris, PUF, 2004.

19. Notice de Jean-Maurice Monnoyer à «L'ÆEuvre d'art à l'époque de sa reproduction mécanisée ", in Walter Benjamin, Écrits français, Paris, Gallimard, 2006, p. 149.

20. Sur le cinéma comme expérience du voyage et la « géographie » de l'affect, de l'émotion et du sentiment, voir Giuliana Bruno, Atlas of Emotion. Journeys in Arts, Architecture and Film, London/ New York, Verso, 2007. 
21. Jean-François Lyotard, Discours, Figure, Paris, Klincksieck, 1971, p. 191 ; Jacques Boulet, « Les appareils de l'architecture. La projection orthogonale », in Jean-Louis Déotte (dir.), Appareils et formes de la sensibilité, Paris, L'Harmattan, 2005.

22. Voir Gérard Conio, Eisenstein. Le cinéma comme art total, Gollion, Infolio, 2007.

23. Frances A. Yates, L'art de la mémoire, Paris Gallimard, 1975.

\section{RÉSUMÉS}

La ville à l'écran est le substrat imaginaire et historique du cinéma. Mais le cinéma, burlesque et soviétique, transforme ce contexte tout en constituant sa mémoire. Walter Benjamin inaugura cette problématique avec la notion de « distraction », liant architecture et cinéma sur le fond du paradigme de la stratification de l'appareil psychique chez Freud.

\section{INDEX}

Personnes citées : Benjamin (Walter), Chaplin (Charlie), Darwin (Charles), Eisenstein (Sergueï), Freud (Sigmund), Kant (Emmanuel), Kracauer (Siegfried), Lefebvre (Henri), Leroi-Gourhan (André), Lyotard (Jean-François), Vertov (Dziga)

Mots-clés : affect, appareil, appareil cinématographique, appareil psychique, architectonique, architecture, aura, burlesque, choc, distraction, espace, expérience, flâneur, habitude, inconscient visuel, masses, mémoire, montage, perception, perception optique, perception tactile, perception urbaine, simultanéité, soviétique, stratification, support, technique, ville, vitrine

\section{AUTEUR}

\section{PASCAL ROUSSE}

Architecte et doctorant en philosophie 